\title{
Phenomenological analysis of elastic scattering reactions using different models
}

\author{
R. I. Badran* \\ Physics Department, King Abdulaziz University, Jeddah, Kingdom of Saudi Arabia and \\ Physics Department, The Hashemite University, P.O. Box 150459, Zarqa, Jordan \\ H. Badahdah \\ Physics Department, King Abdulaziz University, Jeddah, Kingdom of Saudi Arabia
}

(Received on 17 August, 2009)

\begin{abstract}
McIntyre and Frahn-Venter three-parameter models are used to analyze the experimental data of three elastic scattering reactions $\alpha+{ }^{58} \mathrm{Ni}, \alpha+{ }^{116} \mathrm{Sn}$ and $\alpha+{ }^{197} \mathrm{Au}$ at the same incident energy of $240 \mathrm{MeV}$. The different $\alpha$-scattering cases have the same number of minima and maxima in their oscillatory structures of angular distribution. The increase in the mass of target nucleus leads to a smaller nuclear deflection minimum and causes the corresponding angular distribution to become smoother and with steeper slope. The Coulomb damping of Fraunhofer oscillations has an effect accompanied with the increase of mass of target nucleus. The presence of semi-classical phenomena such as Fresnel and Fraunhofer diffraction patterns etc., has been found by analyzing the experimental data of elastic scattering reactions, ${ }^{16} \mathrm{O}+{ }^{64} \mathrm{Zn},{ }^{32} \mathrm{~S}+{ }^{64} \mathrm{Ni}$, and ${ }^{58} \mathrm{Ni}+{ }^{27} \mathrm{Al}$ at several laboratory energies. The generalised Fresnel model can fairly reproduce the angular distribution of these reactions. Excellent fitting can be obtained using Regge pole model, especially at backward angles where the data can not be recovered by the other three models. The adopted theoretical models can reasonably account for the general pattern of the data, thus allowing us to extract important parameters from elastic scattering processes. The analysis also shows that the total reaction cross section has an energy-dependent trend similar to that found by other models.
\end{abstract}

Keywords: Heavy-ion scattering; Regge pole analysis; Strong absorption models

\section{INTRODUCTION}

In heavy-ion collisions, the absorption flux from the elastic channel and the corresponding feeding of other reaction channels are usually very strong. In other words, if the impact parameter of incident particles is smaller than a critical value, the corresponding partial-wave contribution to the elastic scattering amplitude is strongly damped. In several nuclear scattering cases, the incident particle is strongly absorbed upon entering the target nucleus. One of the main advantages of elastic scattering in the presence of strong absorption is that the experimental data can be described without any knowledge about the details of the absorption mechanism. In relatively light systems, especially with closed or semi-closed shell nuclei, absorption is weaker, and both diffractive and refractive effects in elastic scattering can be observed. In semiclassical language, different trajectories may contribute to the total scattering amplitude at the same angle. In the near-sidefar-side decomposition model, one considers trajectories originating from different sides of the scattering potential, and the interference between them leads to Fraunhofer diffraction at forward angles. The interference between two far-side components leads instead to rainbow scattering. These refracting effects are visible at Airy minima in the angular distributions. The appearance of rainbow scattering in the experimental data is possible when the nuclear potential is strong enough (deep real part) to deflect particles into negative angles and when the absorption is incomplete (shallow imaginary part). The partial wave expansion gives us the amplitude of the scattered wave in the asymptotic region far away from the scattering potential. The scattering matrix elements $S_{\ell}$ represent

*Electronic address: rbadran@kau.edu.sa the accumulative effect of the scattering potential on the incident wave. Their determination requires therefore detailed knowledge of the corresponding partial wave function in the interaction region. This, in turn, requires solving Schrodinger equation for some assumed form of interaction potential. On the other hand, $S_{\ell}$ can be determined experimentally by a partial wave fitting to the measured elastic scattering. Matrix elements determined in this way can then be compared with values predicted by theoretical calculations. The determination of $S_{\ell}$ provides therefore a very effective way of comparing theories of elastic scattering with experiment, as these elements are directly related to the measurable crosssections, on the one hand, and to the asymptotic form of the scattering wave function on the other. Moreover, the knowledge of interaction radii, critical angular momenta, surface diffusenesses, and more detailed features of the effective ionion potential are needed as input for the theoretical description of quasielastic reactions. The equivalents of the interaction radii and the surface diffusenesses in angular momentum space are the grazing angular momenta and the angular momentum window width. The first parameter affects the number of oscillations in the angular distribution and the second has an effect on the fall off of the envelope of oscillations of angular distribution. Such effects may be described by a function defined by the Fourier transform of an absorptive shape function. In the presence of strong absorption, several theoretical investigations were based on numerical and analytical treatments of the partial wave expansion model for elastic scattering [1-14]. Most of these investigations showed clearly how sensitive the scattering data for strongly absorbed particles are to the detailed form of the scattering matrix around the grazing angular momentum. Some of these investigations used the extracted McIntyre parameters of the elastic scatterings for the entrance and exit channels of transfer reactions [10-12]. Other investigations demonstrated a wide scope of the use of the strong absorption model in explaining the elas- 
tic scattering of heavy ions [15]. These investigations also exposed and resolved the presence of radius anomaly. However the use of strong absorption model was also extended to low energy heavy-ion elastic scattering [16].

The objective of this work is to analyse experimental data of $\alpha$ particles scattered by different target nuclei of ${ }^{58} \mathrm{Ni}$, ${ }^{116} \mathrm{Sn}$ and ${ }^{197} \mathrm{Au}$ at the same incident energy of $240 \mathrm{MeV}$ [17] on the basis of strong absorption model. Both the numerical model of McIntyre and the analytical treatment of Frahn and Venter are employed. The quality of the fits and correlation between the parameters extracted from analysis of both models are demonstrated. These models are used to explain the presence of semi-classical phenomena such as Fresnel and Fraunhofer diffraction patterns etc... in the angular distribution of elastic scattering reactions. The threealpha scattering cases with the same incident energy exhibit the same number of minima and maxima in their oscillatory structure. However, the angular distribution becomes smoother and with steeper slope as the mass of target nucleus increases. This explains the enhancing effect of Coulomb damping of Fraunhofer oscillations which is controlled by the product $\left(\Delta \theta_{R}\right)$ of surface diffusivity and the Rutherford grazing angle and therefore increases (almost exponentially) with increasing Coulomb parameter and surface diffusivity $\Delta$. The theoretical results of angular distributions obtained from both models using three parameters are fairly compared to experimental data and to the results obtained by the modified Coulomb Glauber model [18].

The analysis of the experimental data of elastic scattering reactions of ${ }^{16} \mathrm{O}+{ }^{64} \mathrm{Zn}$ at laboratory energies 40, 41, 42.5, $43.5,44,48,52,54,56,62$ and $64 \mathrm{MeV}[13,19],{ }^{32} \mathrm{~S}+{ }^{64} \mathrm{Ni}$ at laboratory energies 82, 88, 91, 93, 98, 108 and $150 \mathrm{MeV}$, and ${ }^{58} \mathrm{Ni}+{ }^{27} \mathrm{Al}$ at laboratory energies $155,160,170,185$ and $220 \mathrm{MeV}[14,20,21]$ using both Frahn-Venter model and the generalised Fresnel model is another objective. It is found that such models reproduce reasonably most of the experimental data of these scattering reactions. Regge pole analysis succeeds to give excellent agreement with experimental data of ${ }^{16} \mathrm{O}+{ }^{64} \mathrm{Zn}$ scattering at the energies 40, 41, 42.5, and $43.5 \mathrm{MeV}$, and especially at backward angles where other models fail. The theoretical fittings of measured angular distributions obtained, here, using the strong absorption model of Frahn and Venter with two and three parameters, are compared to the theoretical results of others using the numerical model of McIntyre with three parameters [13, 14].

\section{THEORETICAL BACKGROUND}

\subsection{McIntyre Model}

The nuclear scattering matrix elements $S_{\ell, N}$ may be written as:

$$
S_{\ell, N}=\eta_{\ell} e^{2 i \delta_{\ell, N}}
$$

$S_{\ell, N}$ are expressed in terms of the modified sharp cut-off reflection function $\eta_{\ell}$ and phase shifts $\delta_{\ell, N}$. Here, the concept of nuclear phase shifts $\delta_{\ell, N}$ is introduced into the partial wave to account for the effect of nuclear potential in the nuclear scattering. Moreover, $\eta_{\ell}$ may have a smooth transition, in $\ell$ space, when the partial wave changes from zero for small $\ell$ to unity for large $\ell$ around grazing angular momentum value $\ell_{g}$ and over a range of $\Delta_{M}$. The modified reflection coefficient function is given by the parameterized form [7-8]:

$$
\eta_{\ell}=\left(1+e^{-\left(\ell-\ell_{g}\right) / \Delta_{M}}\right)^{-1}
$$

The phase shifts $\delta_{\ell, N}$ increase when the partial wave $\ell$ decreases and vanishes when $\ell$ becomes large enough such that it does not approach the target nucleus. The phase shifts $\boldsymbol{\delta}_{\ell, N}$ may have the parameterized form given as $[7-8,10]$ :

$$
\delta_{\ell, N}=\mu_{M}\left(1+e^{\left(\ell-\ell_{g}^{\prime}\right) / \Delta_{M}^{\prime}}\right)^{-1} .
$$

Five adjustable parameters $\ell_{g}, \Delta_{M}, \ell_{g}^{\prime}, \Delta_{M}^{\prime}$ and $\mu_{M}$ appear in equations 2 and 3. Here, $\ell_{g}$ and $\ell_{g}^{\prime}$ are grazing angular momenta in $\ell$-space which are semiclassically related to the interaction radius of the colliding nuclei in real space. The diffusivity parameters $\Delta_{M}$ and $\Delta_{M}^{\prime}$ measure the width of transition region in momentum space. The parameter $\mu_{M}$ is necessary to introduce the strength of the nuclear phase shift and causes to affect the amplitude of oscillations of angular distribution. It may also have an effect on steepening the average slope of the envelope of angular distribution.

The five adjustable parameters in this model can be reduced to three parameters when the assumption is made that $\ell_{g}=\ell_{g}^{\prime}$ and $\Delta_{M}=\Delta_{M}^{\prime}$. The expression for the parameter $\Delta_{M}$, can be written as

$$
\Delta_{M}=k d\left(1-\frac{n}{k R}\right)\left(1-\frac{2 n}{k R}\right)^{-1 / 2} .
$$

This indicates that particles moving along classical orbits and penetrating the diffuse surface region of nuclear density will be only partially absorbed. Here, $n$ is the Sommerfeld parameter. The corresponding diffusivity parameter to $\Delta_{M}$ in real space is $d$.

The semiclassical relation between grazing angular momentum $\ell_{g}$ and the interaction radius $R$ can be expressed by:

$$
\ell_{g}+\frac{1}{2}=k R\left(1-\frac{2 n}{k R}\right)^{1 / 2}
$$

The $\frac{1}{2}$ term can be dropped for $\ell_{g}>>1$, which is usually fulfilled in heavy-ion scattering above Coulomb barrier.

The nuclear scattering amplitude in the partial wave analysis has the expression:

$$
f_{N}(\theta)=(2 i k)^{-1} \sum_{\ell=0}^{\infty}(2 \ell+1) e^{2 i \sigma_{\ell}}\left[S_{\ell, N}-1\right] P_{\ell}(\cos \theta)
$$

Here, $\sigma_{\ell}, P_{\ell}(\cos \theta)$ and $k$ are Coulomb phase shifts, Legendre polynomials and wave number, respectively.

The elastic scattering cross section is given by the square modulus of the elastic scattering amplitude $f(\theta)$ for the scattering of nonidentical spin-zero spherical particles. However, $f(\theta)$ is composed of two parts, namely, the Coulomb scattering amplitude $f_{R}(\theta)$ and the nuclear scattering amplitude $f_{N}(\theta)$. 


\subsection{Frahn-Venter Model (FVM)}

In this model, the smooth variation of $\eta_{\ell}$ and $\delta_{\ell}$, introduced by McIntyre [7-8], was represented by a differentiable function of the continuous variable $\lambda=\ell+\frac{1}{2}$, and the summation over $\ell$ in the partial wave expansion was replaced by an integration over $\lambda$. In other words, the diffraction arising from the smoothed edge of the reflection function $S_{\ell}$ near the grazing angular momentum $\ell_{g}$ was replaced by the scattering function $S(\lambda)=S_{N}(\lambda) e^{2 i \sigma(\lambda)}$ near the grazing value of $\lambda$, (i.e. $\Lambda=\ell_{g}+\frac{1}{2}$ ). Here $\sigma(\lambda)$ is the Coulomb phase shift and the nuclear scattering function $S_{N}(\lambda)\left(=\eta(\lambda) e^{2 i \delta_{N}(\lambda)}\right)$ is defined in terms of the continuous angular momentum variable $\lambda$. $\eta(\lambda)$ is the reflection coefficient function which changes rapidly from zero to unity over the transition region centred around the grazing angular momentum $\Lambda$, and $\delta_{N}(\lambda)$ is the nuclear phase shift function. The scattering amplitude, $f(\theta)$, as given by the closed formalism [1-3], is composed of two components, namely, $f(\theta)=f^{+}(\theta)+f^{-}(\theta)$. The component $f^{+}(\boldsymbol{\theta})$ consists of the Rutherford scattering amplitude $f_{R}(\boldsymbol{\theta})$ plus a nuclear amplitude part which depends on the Fourier transform of the absorptive shape function $D_{N}(\lambda)=\frac{d S_{N}(\lambda)}{d \lambda}$. The component $f^{-}(\theta)$ consists of a nuclear part only and depends also on the Fourier transform of the absorptive shape function $D_{N}(\lambda)$ with variable $\theta-\theta_{R}$ instead of $\theta_{R}-\theta$. In this manner the closed formalism formally divides the scattering angles into two main regions. The first is an illuminated region, $\theta<\theta_{R}$, where the main contribution is from Rutherford scattering. The second is a shadow region, $\theta$ $>\theta_{R}$, which corresponds to absorbed Coulomb trajectories. The Fourier transforms of $D_{N}(\lambda)$, which is localised in the $\lambda$-space around $\Lambda$, transform this function into the conjugate space of scattering angles $\pm\left(\theta_{R}-\theta\right)$ centred around $+\theta_{R}$ and $-\theta_{R}$, respectively. However, these transforms represent two diffracted waves, one centred around the near-side grazing trajectory $\theta=+\theta_{R}$ and the other around the far side trajectory $\theta=-\theta_{R}$. The diffraction is generated, therefore, by two effective windows in the $\lambda$-space, one on each side of the nucleus.

The scattering in the illuminated region is essentially produced by Coulomb trajectories on which interference from near-side nuclear diffraction is superimposed. However the main contribution, for angles in the shadow region, arises from the near-side nuclear diffraction whose amplitude falls off as $\theta$ increases above $\theta_{R}$. Interference with far-side nuclear diffraction will produce Fraunhofer-type oscillations. These oscillations are damped by the presence of residual Coulomb scattering in the shadow region which, in turn, depends on the diffusivity $\Delta$. On the other hand, Fraunhofer diffraction can be enhanced by an increase of the refractive effects of the attractive nuclear potential near the surface. In classical terms, the attractive nuclear potential reduces the effect of Coulomb repulsion and brings the Coulomb trajectories in the grazing region towards the forward direction. This, in turn, increases the overlap between the two diffracted waves, thus enhancing the Fraunhofer oscillations.

In the present analysis, the Woods-Saxon-type of reflection coefficient similar to that in equation 2 , is used when the variables $\lambda, \Lambda$ and $\Delta$ replace $\ell, \ell_{g}$ and $\Delta_{M}$, respectively [2]. However, the assumption is made such that three ad- justable parameters are found important, namely, $\Lambda, \Delta$ and $\mu$. The above-mentioned features of heavy-ion collisions result in characteristic qualitative features, which are observed in measured angular distributions of elastic scattering. The observed angular distributions are mainly determined by the interplay between the (quantal) diffractive effects of strong absorption and the (semi-classical) refractive effects produced by the repulsive long range Coulomb field and the attractive, short range nuclear field.

The type of diffraction pattern from a target nucleus with effective radius $R$ is characterised, as in classical optics, by a parameter $p$ which is defined as the ratio of the Rayleigh length (i.e. the length of the shadow behind the target) and the shorter of the distances of the observation point or of the source point from the centre of the target. In our case, the analogy of this "pattern parameter" is $p=\frac{2 n}{1+(n / \Lambda)^{2}}$ where its value is determined by Sommerfeld parameter $n$ and the grazing angular momentum parameter $\Lambda$. For $p<<1$ the diffractive Fraunhofer-type dominates the angular distribution of elastic scattering and for $p>1$ the Fresnel-type scattering is dominant.

A simple classification to all scattering processes of charged particles above the Coulomb barrier [4] may be realised by knowing both Coulomb parameter $n$ and the ratio, $h$. Here, the ratio of centre of mass kinetic energy $E_{c . m}$. to the Coulomb barrier $V_{C}$, is expressed as

$$
h=\frac{1}{2}\left(1+\csc \frac{\theta_{R}}{2}\right) .
$$

\subsection{Generalized Fresnel Model (GFM)}

The generalized Fresnel model (GFM) provided a simple closed formula for the ratio of the differential elastic scattering cross section to Rutherford cross section [5, 6]. This allowed for easy extraction of the grazing angular momentum and the width of angular momentum in $\lambda$-space with the least time of computation and then, in turn, determining the total reaction cross section. This method was successful for light projectiles (like ${ }^{16} \mathrm{O}$ and ${ }^{20} \mathrm{Ne}$ ) [6] as well as for very heavier projectiles (like ${ }^{84} \mathrm{Kr}$ ) on heavy targets [6]. It was shown that in the Coulomb limit and after conducting some mathematical simplifications to the analytical formula of scattering amplitude of Frahn and Venter, a modified form of the ratio between the elastic scattering amplitude to Rutherford amplitude was resulted [6], namely,

$$
\frac{f(\theta)}{f_{R}(\theta)}=1-\frac{1}{2} \operatorname{erfc}\left(-e^{\frac{1}{4} i \pi} u\right) F\left[\Delta\left(\theta_{R}-\theta\right)\right],
$$

in the illuminated region at $\theta<\theta_{R}$, and

$$
\frac{f(\theta)}{f_{R}(\theta)}=\frac{1}{2} \operatorname{erfc}\left(e^{\frac{1}{4} i \pi} u\right) F\left[\Delta\left(\theta_{R}-\theta\right)\right],
$$

in the shadow region at $\theta>\theta_{R}$. Here, $u$ is defined by the expression

$$
u=\left(\theta-\theta_{R}\right)\left(\frac{\Lambda}{2 \sin \theta_{R}}\right)^{\frac{1}{2}}
$$


The function $F\left[\Delta\left(\theta_{R}-\theta\right)\right]$ is the Fourier transform of the absorptive shape function $D_{N}(\lambda)$ and is given by $[1,16]$ :

$$
F=\frac{\pi \Delta\left(\theta_{R}-\theta\right)}{\sinh \left[\pi \Delta\left(\theta_{R}-\theta\right)\right]} \text {. }
$$

Here $\theta_{R}$ is the Rutherford grazing angle given by $\theta_{R}=$ $2 \tan ^{-1}\left(\frac{n}{\Lambda}\right)$. The interaction radius and the diffusivity are given by $[1,16]$ :

$$
\begin{aligned}
& k R=n+\sqrt{\Lambda^{2}+n^{2}}, \\
& k d=\frac{\Delta}{\sqrt{1+(n / \Lambda)^{2}}}
\end{aligned}
$$

The GFM formulae, in equations 8 and 9 , are valid when the Coulomb interaction plays a dominant role. Here, the three adjustable parameters used in Frahn-Venter model $(\Lambda$, $\Delta$ and $\mu$ ) are reduced into two parameters only, namely, $\Lambda$ and $\Delta$.

A more simplified model was also used to analyze several elastic scattering using a single parameter, that is, $\Lambda$ [6]. This is called the Fresnel model (FM) [6].

\subsection{Regge Pole Model (RPM)}

The scattering matrix elements are modified due to the presence of large transparency associated with deep real potential which gives rise to Regge poles. The parameterized scattering matrix $S^{\text {Regge }}(\lambda)$ is written as [22]:

$$
S^{\text {Regge }}(\lambda)=S(\lambda)+\frac{i D(\lambda) e^{i \phi_{\lambda_{o}}}}{\lambda-\lambda_{o}-\frac{i \Gamma(\lambda)}{2}} .
$$

The amplitude $\mathrm{D}(\lambda)$ and the width $\Gamma(\lambda)$ of the pole are represented as damping functions of the forms:

$$
\begin{aligned}
& D(\lambda)=D_{o}[1-\operatorname{ReS}(\lambda)], \\
& \Gamma(\lambda)=\Gamma_{o}[1-\operatorname{ReS}(\lambda)] .
\end{aligned}
$$

Here $S(\lambda)$ is given by the relation:

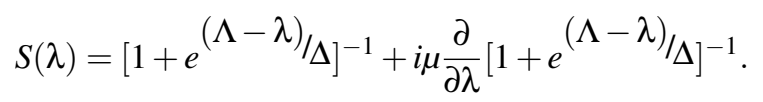

The parameters $\phi_{\lambda_{o}}$ and $\lambda_{o}$ are the phase and orbital angular momentum of the pole.

The semiclassical relation between grazing angular momentum, $\Lambda$, and the interaction radius, $\mathrm{R}$, is same as that in equation 5 with $\Lambda=\ell_{g}+\frac{1}{2}$. Moreover, the diffusivity, $\Delta$, in $\lambda$-space is expressed in similar manner to $\Delta_{M}$ (equation 4 ).

The interaction radius $R$ is defined by:

$$
R=r_{0}\left(A_{P}^{1 / 3}+A_{T}^{1 / 3}\right) .
$$

The quantities $A_{P}$ and $A_{T}$ are the atomic masses of projectile and target nuclei, respectively, while $r_{o}$ is an adjustable parameter.

Here, Regge pole analysis is conducted using seven parameters $r_{o}, d, \mu, \phi_{\lambda_{o}}, \lambda_{o}, D_{o}$, and $\Gamma_{o}$.

\section{RESULTS AND DISCUSSION}

FORTRAN codes are developed to calculate the crosssections of elastic scattering reactions at different angles and energies using McIntyre, Frahn-Venter, Regge pole and generalized Fresnel models. The best fit to the experimental data of angular distribution of elastic scattering is obtained and the final choice of extracted parameters is considered when the value of $\chi^{2}$ is minimum for each energy. The average value of $10 \%$ of the experimental data is considered for each experimental error at a certain energy of an elastic scattering. However, each of the adjustable parameters $r_{o}, d$ and $\mu$ for McIntyre model or each of the adjustable parameters $(\Lambda, \Delta$ and $\mu$ ) for Frahn and Venter model, or, $(\Lambda, \Delta)$ for the generalised Fresnel model or each of the adjustable parameters $r_{o}$, $d, \mu, \phi_{\lambda_{o}}, \lambda_{o}, D_{o}$, and $\Gamma_{o}$ for Regge pole model is introduced with an initial value and is changed in very small steps. The codes are developed to allow for all possible combination of parameters that give fits to the experimental data. The iterations establish a set of values for the adjustable parameters and the final choice is made with the best fit when the quality of fit is judged by $\chi^{2}$ indicator. The theoretical curves of angular distribution will be fitted, using both models, to the corresponding experimental data when the value of $\chi^{2}$ is minimum.

\subsection{Elastic Scattering of $\alpha+{ }^{58} \mathrm{Ni}$}

The calculated angular distribution for $240 \mathrm{MeV}$ alpha particles on ${ }^{58} \mathrm{Ni}$, using both models, is shown in Figure 1, together with the experimental data [17]. The theoretical curve represents the best fit to experimental data and is obtained using the adjustable parameter values of $r_{0}=1.18 \mathrm{fm}, d=0.46 \mathrm{fm}$ and $\mu=0.95 \mathrm{rad}$, when McIntyre model is used. The theoretical results compare well with those obtained using Coulomb modified Glauber CMG model with set B [21]. The obtained set of parameters is listed in Table 1 . The value of radius parameter $\left(r_{0}=1.18 \mathrm{fm}\right)$ obtained for alpha particles on ${ }^{58} \mathrm{Ni}$ is expected to be smaller than value of $1.55 \mathrm{fm}$ for heavy ions. The reason for this is attributed to the tight structure of the alpha-particles. The formula for the strong interaction radius $\left[R=r_{0}\left(A_{1}^{1 / 3}+A_{2}^{1 / 3}\right)\right]$ is not expected to hold for the alphaparticles, but can be rewritten in the form $R=r_{0} A_{2}^{1 / 3}+r_{\alpha}$. Using reasonable value of $r_{\alpha}=1.45 \mathrm{fm}$ [23-24] and the strong absorption radius for $\alpha+{ }^{58} \mathrm{Ni}$ obtained from the present analysis $(6.44 \mathrm{fm})$, yield a value of $r_{0}=1.29 \mathrm{fm}$. From a similar analysis of this scattering process but at $43 \mathrm{MeV}$ laboratory energy, a value of $1.36 \mathrm{fm}$ is rather obtained from the fitting which becomes $1.5 \mathrm{fm}$ when the above value of $r_{\alpha}$ is used instead [11]. Figure 1 also shows the theoretical results of cross-section obtained from employing FVM. The results of this model are in good agreement with those of McIntyre model especially at angles less than $10^{\circ}$. The set of parameters extracted for this scattering using FVM is also listed in Table 1. A value of $r_{0}(=1.38 \mathrm{fm})$, which is not so far from $1.55 \mathrm{fm}$, is obtained. The value of strong absorption radius for the same reaction is found $(\mathrm{R}=6.81 \mathrm{fm})$ using this latter model. 


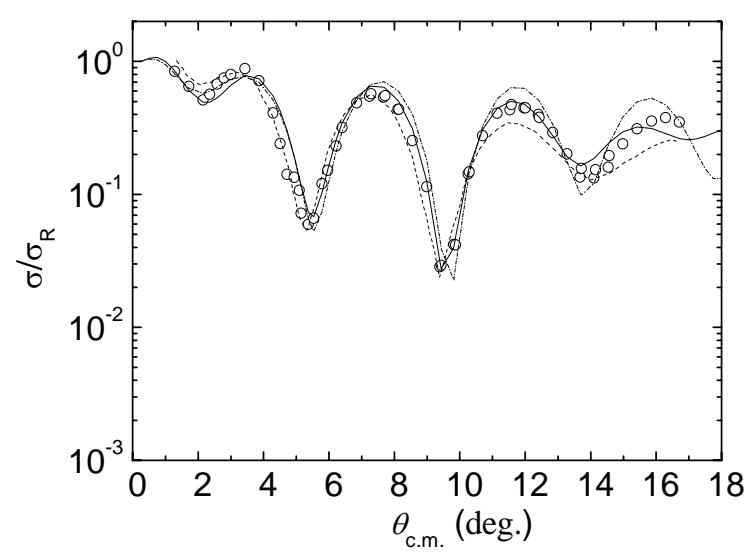

FIG. 1: The measured angular distribution (symbols) of elastic scattering $\alpha+{ }^{58} \mathrm{Ni}$ at $E_{\text {lab }}=240 \mathrm{MeV}$ [17]. The theoretical results of angular distribution obtained using McIntyre (dash-dotted line) and Frahn-Venter (solid line) models. The dashed curve represents the results of CMG model using set B [18].

\subsection{Elastic Scattering of $\alpha+{ }^{116} S n$}

The experimental data of angular distribution for $\alpha+{ }^{116} S n$ at $240 \mathrm{MeV}$ laboratory energy [17] is fitted using both McIntyre and Frahn-Venter models. The experimental data and theoretical results are in good agreement and are shown in Figure 2. The theoretical results obtained from both models agree remarkably with each other. The theoretical results of CMG model using set B [18] together with those of optical model [17] are also presented. Our results show better agreement to both the experimental data and theoretical results of optical model than those of CMG model.

The values of adjustable parameters and other useful physical parameters together with the values of $\chi^{2}$, using both McIntyre and Frahn-Venter models, are listed in Table 1. The values of the parameters extracted from McIntyre model for $r_{0}$ and $d$ have slight increase over those obtained for previous scattering process using the same model. Consequently the ratio $d / R$ exhibits almost similar behavior, although $r_{0}$ and $d$ differ slightly in the two models. From both models the obtained value of $\Delta$ exhibits a trend that agrees very well with the prescriptions of the strong absorption model.

The diffraction pattern, from both models, can be shown to be consistent with that of the experimental data, as long as the period and amplitude of the oscillation are concerned.

\subsection{Elastic Scattering of $\alpha+{ }^{197} A u$}

Figure 3 shows the calculated angular distribution for 240 MeV alpha particles by ${ }^{197} A u$, using both McIntyre and Frahn-Venter models, together with the corresponding experimental data. The theoretical results of CMG model using set B, [18] together with those of optical model [17] are also presented. Our results compare much better, to both the experimental data and theoretical results of optical model, than those of CMG model.

The best fit is obtained, using the adopted models, with the

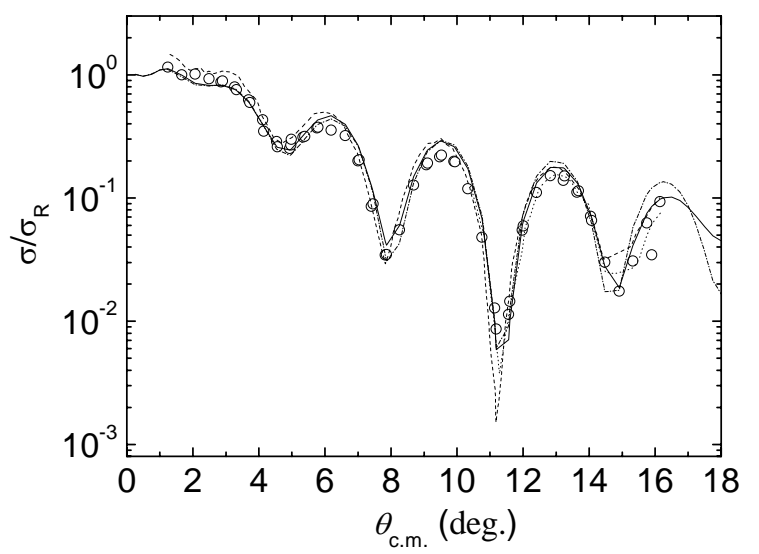

FIG. 2: The measured angular distribution (symbols) of elastic scattering $\alpha+{ }^{119} \mathrm{Sn}$ at $E_{l a b}=240 \mathrm{MeV}$ [17]. The theoretical results of angular distribution obtained using a) McIntyre model (dash-dotted line) and b) Frahn-Venter model (solid line). The dashed curve represents the results of CMG model using set B [18]. The dotted curve is the theoretical results of optical model [17].

values of $\chi^{2}$ listed in Table 1 . The parameters of the elastic scattering are also listed in this table. The value of $r_{0}=1.23$ fm obtained from the analysis of this scattering employing McIntyre model is a little bit larger than that obtained for the previous scattering processes $\left(\alpha+{ }^{58} N i, \alpha+{ }^{116} S n\right)$. However, the values of $d=0.48 \mathrm{fm}$ and $\Delta_{M}=3.19$ for this scattering process are found larger than those obtained for the previous scattering processes (i.e. $\alpha+{ }^{58} N i$ and $\alpha+{ }^{116} S n$ ), as well. Moreover, the analysis of FVM also gives a similar trend of increase in the values of the parameters $d$ and $\Delta$ when the target nucleus gets larger. This means that the larger is the target nucleus, the wider is the width of the window of angular momentum in $\ell$-space when the incident energy is fixed. This is to allow for larger number of partial waves to pass through the surface of target nucleus. The ratio $d / R$ monotonically decreases with the increase in the size of target nucleus, in both models. Moreover, the value of $\mu$ for this scattering, due to both models, decreases when the target nucleus ${ }^{197} \mathrm{Au}$ replaces ${ }^{58} \mathrm{Ni}$ or ${ }^{116} \mathrm{Sn}$. This decrease is consistent with the presence of shallower oscillations, exhibited by the experimental data of angular distribution. The exponential fall off of experimental data of the angular distribution, at $\theta>\theta_{R}$, gives a value of $\Delta$ agreeable with that extracted from McIntyre model.

The quality of the theoretical fit to measured angular distribution is found to be sensitive to the choice of parameters in both models. Small changes in any of the three adjustable parameters produced marked disagreement with the experiment in the direction expected on the basis of the strong absorption prescriptions. In McIntyre model, for example, the decrease in the value of $r_{o}$ may be resulted in pushing out the Fraunhofer oscillation to higher scattering angles. The increase in the value of the parameter $d$ may cause an increase in the slope of the envelope of the calculated Fraunhofer oscillations. The increase in the value of the nuclear phase parameter $\mu_{M}$ has its effect on enhancing the diffraction structure of the calculated angular distribution. This is 


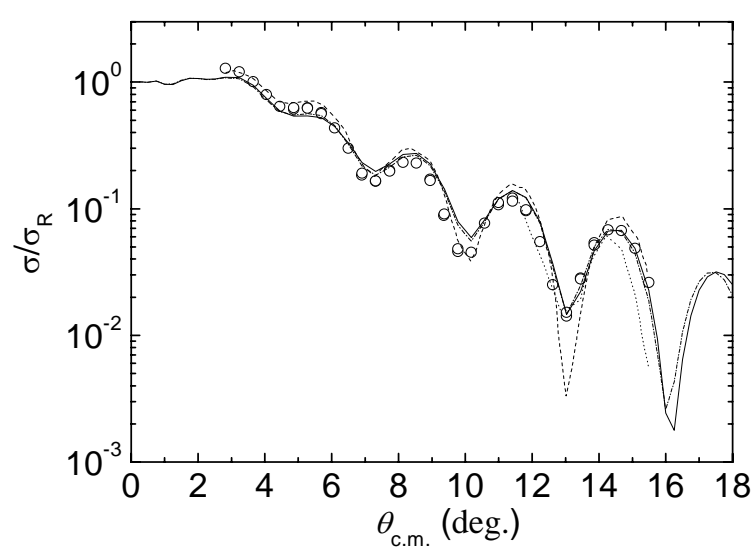

FIG. 3: Same as in figure 2, but for elastic scattering $\alpha+{ }^{197} A u$ at $E_{l a b}=240 \mathrm{MeV}$.

\begin{tabular}{|c|c|c|c|}
\hline Elastic scattering & $\alpha+{ }^{58} \mathrm{Ni}$ & $\alpha+{ }^{116} \mathrm{Sn}$ & $\alpha+{ }^{197} \mathrm{Au}$ \\
\hline $\begin{array}{c}r_{0}(\mathrm{fm})[\text { McIntyre }] \\
{[\mathrm{FVM}]}\end{array}$ & $\begin{array}{l}1.18 \\
1.25\end{array}$ & $\begin{array}{l}1.22 \\
1.26\end{array}$ & $\begin{array}{l}1.23 \\
1.26\end{array}$ \\
\hline $\begin{array}{c}\mathrm{d}(\mathrm{fm}) \text { [McIntyre] } \\
{[\mathrm{FVM}]}\end{array}$ & $\begin{array}{l}0.46 \\
0.36 \\
\end{array}$ & $\begin{array}{l}0.47 \\
0.37 \\
\end{array}$ & $\begin{array}{l}0.48 \\
0.42 \\
\end{array}$ \\
\hline $\begin{array}{c}\mu \text { (rad) [McIntyre] } \\
{[\text { FVM] }}\end{array}$ & $\begin{array}{c}0.95 \\
4.2\end{array}$ & $\begin{array}{l}0.9 \\
3.8\end{array}$ & $\begin{array}{c}0.60 \\
3.2\end{array}$ \\
\hline$\mu / 2 \Delta$ & 0.91 & 0.79 & 0.57 \\
\hline $\begin{array}{l}\ell_{g}[\text { McIntyre }] \\
\Lambda \quad[\text { FVM }]\end{array}$ & $\begin{array}{l}39 \\
42\end{array}$ & $\begin{array}{c}49 \\
51.5\end{array}$ & $\begin{array}{c}59 \\
58.6\end{array}$ \\
\hline $\begin{array}{c}\Delta \text { [McIntyre }] \\
{[\text { FVM }]}\end{array}$ & $\begin{array}{c}2.92 \\
2.3\end{array}$ & $\begin{array}{c}3.08 \\
2.4\end{array}$ & $\begin{array}{c}3.19 \\
2.4\end{array}$ \\
\hline $\begin{array}{c}\mathrm{R}(\mathrm{fm}) \text { [McIntyre] } \\
{[\mathrm{FVM}]}\end{array}$ & $\begin{array}{l}6.44 \\
6.81\end{array}$ & $\begin{array}{l}7.89 \\
8.18\end{array}$ & $\begin{array}{l}9.11 \\
9.32\end{array}$ \\
\hline $\begin{array}{c}h \text { [McIntyre] } \\
{[\text { FVM] }}\end{array}$ & $\begin{array}{l}17.93 \\
18.95\end{array}$ & $\begin{array}{l}12.71 \\
13.17\end{array}$ & $\begin{array}{l}9.42 \\
9.63\end{array}$ \\
\hline $\begin{array}{c}\theta_{\mathrm{R}}(\mathrm{rad})[\text { McIntyre }] \\
{[\mathrm{FVM}]}\end{array}$ & $\begin{array}{l}0.058 \\
0.054\end{array}$ & $\begin{array}{l}0.083 \\
0.079\end{array}$ & $\begin{array}{c}0.113 \\
0.11\end{array}$ \\
\hline$\theta_{1 / 4}(\mathrm{rad})$ & 0.077 & 0.086 & 0.117 \\
\hline$\theta_{\text {Nuc. }}(\mathrm{rad})$ & -0.163 & -0.146 & -0.094 \\
\hline$\sigma_{\mathrm{r}}(\mathrm{GFM})(\mathrm{mb})$ & 1453 & 2136 & 2696 \\
\hline$\sigma_{\mathrm{r}}(\mathrm{FM})(\mathrm{mb})$ & 1378 & 1941 & 2444 \\
\hline $\begin{array}{c}\left.\chi^{2} \text { [McIntyre }\right] \\
{[\text { FVM }]}\end{array}$ & $\begin{array}{l}8.11 \\
99.6\end{array}$ & $\begin{array}{c}3.08 \\
4.0 \\
\end{array}$ & $\begin{array}{l}3.33 \\
26.9 \\
\end{array}$ \\
\hline
\end{tabular}

TABLE 1: The extracted parameters for the elastic scattering processes of $\alpha$ particles by the target nuclei ${ }^{58} \mathrm{Ni},{ }^{116} \mathrm{Sn}$ and ${ }^{197} \mathrm{Au}$ at $E_{\text {lab. }}=240 \mathrm{MeV}$ (i.e $60 \mathrm{MeV} / \mathrm{u})$, when McIntyre model is used, are listed.

evidenced by the deepening of the minima of the diffraction oscillations. The increase in the value of $\mu_{M}$ is consistent with the features of strong Fraunhofer oscillations which manifest themselves in the experimental data. In Frahn-Venter model, a corresponding effect to $\mu_{M}$ of McIntyre model, is found approximately equivalent to $\mu / 2 \Delta$ for all of $\alpha$ scattering processes. The three adjustable parameters $(\Lambda, \Delta$ and $\mu)$ from the Frahn-Venter model are also found transparent to the quality of the fit. In particular, any increase in the value of $\Lambda$ may increase the number of oscillations and push the envelope of oscillations to forward angles. However, the increase in $\Delta$ leads to an increase in the slope of envelope of oscillations and dampen them. The increase in the parameter $\mu$ may produce deeper oscillations. The values of $r_{o}=1.21 \pm 0.03 \mathrm{fm}, \mathrm{R}$ $=7.77 \pm 1.33 \mathrm{fm}$ and $\mathrm{d}=0.47 \pm 0.01 \mathrm{fm}$ with corresponding value of $\Delta_{M}=3.06 \pm 0.14$ are obtained for the above mentioned $\alpha$ scattering processes using McIntyre model. Corresponding values of, $\mathrm{R}=8.06 \pm 1.26 \mathrm{fm}$, and $\mathrm{d}=0.39 \pm 0.03$ fm with corresponding value of $\Delta=3.7 \pm 0.5$ are also yielded for the $\alpha$-scattering processes from FVM. There is a mild increase in the value of diffusivity parameter $\Delta$ when the atomic mass of target nucleus increases in both analyses. This is also consistent with the increasing value of $\Delta$ obtained from experimental data of the angular distribution, at $\theta>\theta_{R}$. The decrease in the value of $\mu$ or $\mu_{M}$ with the increase in the atomic mass of target nucleus is experimentally justified by the weaker oscillatory structure. It is interesting to note here that the approximation of $\theta_{R} \approx \theta_{1 / 4}$ starts to weaken when target nucleus becomes lighter. Moreover, the nuclear deflection angle at the grazing angular momentum $\left(\theta_{N u c}\right)$ gets larger when the atomic mass of target nucleus becomes small. All values of the diffraction parameter (i.e. $p>1$ ) assert that all elastic scattering processes exhibits Fresnel-type diffraction pattern. Here, $\Lambda$ and $n$ get larger when the target nucleus atomic mass increases and this leads to a larger diffraction pattern (i.e the value of $p$ increases from 2.27, 4.06 to 6.40 as the target nucleus changes from ${ }^{58} \mathrm{Ni},{ }^{116} \mathrm{Sn}$ and ${ }^{197} \mathrm{Au}$, respectively). The monotonic decrease of the ratio $d / R$ with the increase in the size of target nucleus, in both models, justifies the expected effect of Coulomb damping from these types of scattering processes.

Here, for $\alpha+{ }^{58} \mathrm{Ni}$ the values of $(n, h)=(1.14,18.95)$ or $(\Lambda, p)=(42.0,2.3)$ from Frahn-Venter model (FVM) are comparable to the corresponding values $(n, h)=(1.14,17.93)$ or $\left(\ell_{g}, p\right)=(39.0,2.3)$ from McIntyre model. While for $\alpha+$ ${ }^{116} \mathrm{~S} n$ the values of $(n, h)=(2.03,13.17)$ or $(\Lambda, p)=(51.5$, 4.1) from Frahn-Venter model resemble corresponding values of $(n, h)=(2.03,12.71)$ or $\left(\ell_{g}, p\right)=(49.0,4.1)$ from McIntyre model. The two models give approximately similar values for $(n, h)$ or $(\Lambda, p)$ for the reaction $\alpha+{ }^{197} A u$, as well which enhances the idea of having a unique diffraction pattern. This means that interpretation of the diffraction features is modelindependent.

It is Important to note that the total reaction cross section increases, using both the simple Fresnel model (FM) and the generalized Fresnel model (GFM), with the increase in the mass of target nucleus when the projectile energy remains fixed, as seen in Table 1. The quantity that matters in this analysis is, however, the ratio $\sigma_{r} / \pi R^{2}$ which remains fairly constant around one (i.e. $\sigma_{r} / \pi R^{2}=0.98 \pm 0.02$ ).

\subsection{Elastic Scattering of ${ }^{16} \mathrm{O}+{ }^{64} \mathrm{Zn}$}

The experimental data of angular distribution for the elastic scattering of ${ }^{16} \mathrm{O}$ by ${ }^{64} \mathrm{Zn}$ at eleven energies of 40,41 , $42.5,43.5,44,48,52,54,56,62$ and $64 \mathrm{MeV}$ [19] are presented in Figure 4 . The theoretical results of angular distribution using both the full formalism of Frahn-Venter (FVM) and the generalized Fresnel (GFM) models are also shown in the same figure. The agreement between theoretical and experimental results is quite good when the three-parameter fit is used. The fittings are fairly acceptable using two parameters only (i.e. $\Lambda$ and $\Delta$ ) but for energies $48-64 \mathrm{MeV}$. The fittings become poor, at $\theta>\theta_{R}$, as the incident energy approaches the Coulomb barrier for energies $40-44 \mathrm{MeV}$. It is evident, in most of energies, that the absence of $\mu$ has an effect on the fall-off at $\theta>\theta_{R}$. Table 2 shows the list of parameters used 
for this analysis using both FVM and GFM. It can be easily seen that an increase in incident energy leads to a corresponding increase in $\Lambda$. This also leads to open a wider window for angular momentum of $\Delta$. This corresponding increase of diffusivity $\Delta$ or $d$ in both $\ell$-space or real space, respectively, is found discernible. The average value of $(d=0.12 \pm 0.09 \mathrm{fm})$ or the ratio $d / R$ yielded from our analysis by adopting FrahnVenter model is comparable to a corresponding average value obtained from different analysis based on the strong absorption prescriptions [13]. The reason for the inaccurate estimation of the latter parameter, as compared to the previous cases of scattering processes, may be attributed to the low values of $h$ (i.e. the ratio of the incident centre of mass energy to the Coulomb barrier) in most of the cases. Moreover, the trend of change in $\Delta$ or $d$ starts to depart from the expected prescription of SAM as the incident energy becomes closer to Coulomb barrier. It is worth noting that an average value of strong absorption radius $R=10.5 \pm 0.34 \mathrm{fm}$ is in excellent agreement with corresponding value $R=10.4 \pm 0.35 \mathrm{fm}$ [19] using the optical model. Similar agreement is found with $R=$ $10.35 \pm 0.35 \mathrm{fm}$ using McIntyre model [13]. Both experimental data and theoretical results show that Fresnel-type diffraction is dominant in this set of different energies of scattering process apart from few exceptions. It can also be noted, from Table 2, that the approximation $\theta_{1 / 4} \approx \theta_{R}$ becomes inaccurate when the laboratory energy becomes too low. Here, Regge pole analysis is also conducted using the seven parameters $r_{o}$, $d, \mu, \phi_{\lambda_{o}}, \lambda_{o}, D_{o}$, and $\Gamma_{o}$ for this reaction at energies 40,41 , 42.5 and $43.5 \mathrm{MeV}$. It is evident that this latter model is able to recover the previous unsuccessful reproduction of the data at $\theta>\theta_{R}$, as shown in Figure 4-a. The obtained values of adjustable parameters are listed in Table 3 .

Here, the total reaction cross section has energy-dependent behavior. Figure 5 shows the variation of ratio of total reaction cross-section to $\pi R^{2}$ with the inverse of laboratory energy as obtained from both the Fresnel and generalized Fresnel models. The shown best least-square fittings of the reproduced data enable us to make easy comparison to the slopes of each line obtained from both models.

\subsection{Elastic scattering of ${ }^{32} \mathrm{~S}+{ }^{64} \mathrm{Ni}$}

Figure 6 shows experimental data of ${ }^{32} \mathrm{~S}+{ }^{64} \mathrm{Ni}$ elastic scattering at energies 82, 88, 91, 93, 98, 108 and $150 \mathrm{MeV}$ [21] and the theoretical results using Frahn-Venter model. All parameters obtained from this fit together with the values of $\chi^{2}$ are found in Table 4. The theoretical analysis of angular distribution for this scattering, using Frahn-Venter model, produces features that are consistent with the prescriptions of strong absorption model of Frahn and Venter. The increase in the values of parameters $\Lambda$ and $\Delta$ is correlated to some extent with the increase in incident energy. Some discrepancies from SAM occur when centre of mass energy becomes closer to Coulomb barrier. The decrease in the value of $R$ with the increase in incident energy is also evident. However, the exclusion of $\mu$ in the generalised Fresnel model gives fairly comparable fittings to the experimental data using two parameters only. This means that the generalised Fresnel model can reasonably account for the reproduction of the data in this scattering. The departure from the experimental data and
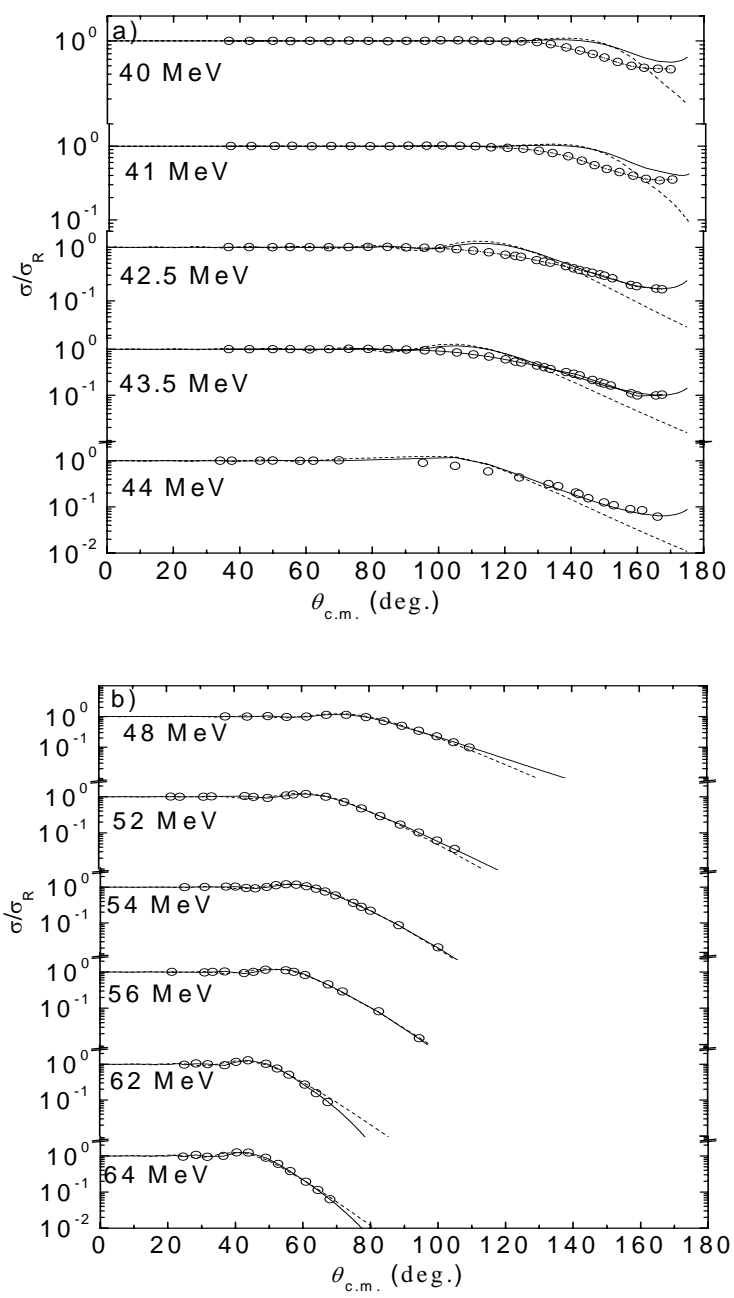

FIG. 4: The measured angular distribution (symbols) of elastic scattering ${ }^{16} \mathrm{O}+{ }^{64} \mathrm{Zn}$ at laboratory energies a) $E_{l a b} .=$ $40,41,42.5,43.5,44 \mathrm{MeV}$, b) $E_{l a b .}=48,52,54,56,62$ and $64 \mathrm{MeV}$ [19]. The theoretical results (solid line) and (dashed line) of angular distribution are obtained using full formalism of Frahn-Venter model (FVM) and the generalized Fresnel model (GFM), respectively. The theoretical results (dash-dotted line) of angular distribution which are reproduced using Regge pole model, appears in Figure 4-a only.

the theoretical results produced from Frahn-Venter model, for most energies, appears at $\theta>\theta_{R}$. Similar conclusions were found for the same scattering from the analysis using McIntyre model [14]. Figure 7 shows the variation of ratio of total reaction cross-section to $\pi R^{2}$ with the inverse of incident energy as obtained from both the Fresnel and generalized Fresnel models. The results from both models start to agree very well at higher energies.

\subsection{Elastic Scattering of ${ }^{58} N i+{ }^{27} \mathrm{Al}$}

The available experimental data [20] of angular distribution for elastic scattering ${ }^{58} \mathrm{Ni}+{ }^{27} \mathrm{Al}$ at laboratory energies 155 , 160, 170, 185 and $220 \mathrm{MeV}$ are analyzed using Frahn-Venter model. Both the theoretical curve and experimental data of 


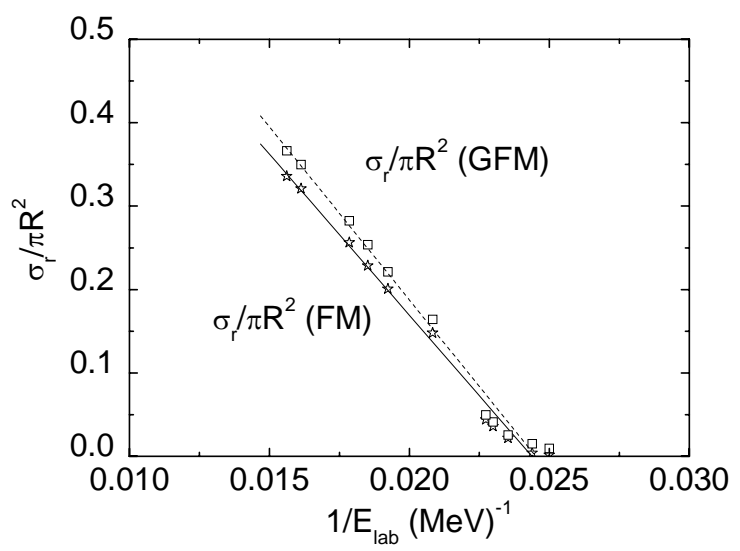

FIG. 5: The variation of the ratio of total reaction cross section to $\pi R^{2}$ with $1 / E_{l a b}$. for the elastic scattering ${ }^{16} \mathrm{O}+{ }^{64} \mathrm{Zn}$ as obtained from using Fresnel (FM) and generalized Fresnel (GFM) models.

\begin{tabular}{|c|c|c|c|c|c|c|c|c|c|c|c|}
\hline$E_{\text {lab. }}(\mathrm{MeV})$ & 40.0 & 41.0 & 42.5 & 43.5 & 44.0 & 48.0 & 52.0 & 54.0 & 56.0 & 62.0 & 64.0 \\
\hline$\Lambda$ & 2.0 & 3.0 & 7.0 & 9.0 & 10.0 & 19.7 & 23.5 & 25.5 & 27.5 & 32.0 & 33.5 \\
\hline$\Delta$ & 1.8 & 2 & 0.55 & 0.6 & 0.6 & 1 & 1.1 & 1.3 & 1.3 & 1.35 & 1.4 \\
\hline$\mu(\mathrm{rad})$ & 0.4 & 0.1 & 0.1 & 0.1 & 0.25 & 0.1 & 0.35 & 0.6 & 0.9 & 1.9 & 1.6 \\
\hline$d(\mathrm{fm})$ & 0.034 & 0.056 & 0.035 & 0.048 & 0.052 & 0.138 & 0.163 & 0.197 & 0.200 & 0.209 & 0.217 \\
\hline$R(\mathrm{fm})$ & 10.82 & 10.58 & 10.39 & 10.30 & 10.27 & 10.56 & 10.39 & 10.37 & 10.37 & 10.25 & 10.17 \\
\hline$h$ & 1.002 & 1.004 & 1.022 & 1.037 & 1.046 & 1.174 & 1.251 & 1.296 & 1.344 & 1.472 & 1.506 \\
\hline$\theta_{R}(\mathrm{rad})$ & 2.975 & 2.889 & 2.555 & 2.393 & 2.315 & 1.673 & 1.457 & 1.358 & 1.267 & 1.081 & 1.040 \\
\hline$\theta_{1 / 4}(\mathrm{rad})$ & --- & --- & 2.669 & 2.511 & 2.417 & 1.716 & 1.476 & 1.368 & 1.269 & 1.064 & 1.030 \\
\hline$\sigma_{\mathrm{r}}(\mathrm{GFM})(\mathrm{mb})$ & 35.03 & 53.41 & 87.02 & 137.0 & 164.9 & 575.3 & 749.5 & 857.2 & 953.9 & 1154 & 1190 \\
\hline$\sigma_{r}(\mathrm{FM})(\mathrm{mb})$ & 6.41 & 14.07 & 73.91 & 119.4 & 145.7 & 518.3 & 680.8 & 771.9 & 865.7 & 1059 & 1090 \\
\hline$\chi^{2}$ & 2.32 & 4.05 & 3.99 & 4.9 & 6.68 & 0.08 & 0.738 & 1.96 & 2.82 & 0.84 & 2.36 \\
\hline$\Lambda(\mathrm{GFM})$ & 2.0 & 3.0 & 7.0 & 9.0 & 10.0 & 19.0 & 23.0 & 25.0 & 27.5 & 32.0 & 33.5 \\
\hline$\Delta(\mathrm{GFM})$ & 1.8 & 2.0 & 0.55 & 0.6 & 0.6 & 1.0 & 1.1 & 1.3 & 1.3 & 1.35 & 1.4 \\
\hline$\chi^{2}(\mathrm{GFM})$ & 2.36 & 3.33 & 7.6 & 9.8 & 7.06 & 0.6 & 0.47 & 0.36 & 0.5 & 1.04 & 0.9 \\
\hline
\end{tabular}

TABLE 2: The parameters for the elastic scatterings of ${ }^{16} \mathrm{O}$ nucleus by the target nucleus ${ }^{64} \mathrm{Zn}$ at incident energy of $40,41,42.5,43.5,44,48,52,54,56,62$ and $64 \mathrm{MeV}$ [19] are found when both the Frahn-Venter model (FVM) and generalized Fresnel model (GFM) are employed. Total reaction cross sections $\sigma_{r}$ (FM) are obtained from Fresnel model (FM).

angular distribution are shown in figure 8. The three parameters obtained from the best fit are listed in Table 5. Both experimental data and theoretical results exhibit very mild oscillations in the shadow and illuminated region. Some oscillations start to appear when the energy increases. This behavior is expected since the decrease of Coulomb effect (or Sommerfeld parameter $n$ ) enhances the Fraunhofer oscillations. This is clearly seen in Table 6 and demonstrated in figure 8 by the

\begin{tabular}{|c|c|c|c|c|}
\hline$E_{\text {lab. }}(\mathrm{MeV})$ & 40.0 & 41.0 & 42.5 & 43.5 \\
\hline$r_{0}$ & 1.659 & 1.621 & 1.578 & 1.56 \\
\hline $\mathrm{d}(\mathrm{rad})$ & 0.056 & 0.088 & 0.21 & 0.28 \\
\hline$\mu(\mathrm{rad})$ & 0.07 & 0.08 & 0.08 & 0.09 \\
\hline$\varphi_{\ell_{0}}$ & 49.5 & 49.5 & 50 & 50 \\
\hline$D_{0}$ & 0.001 & 0.002 & 0.003 & 0.004 \\
\hline$\Gamma_{0}$ & 0.3 & 0.3 & 0.4 & 0.5 \\
\hline$L_{0}$ & 8.8 & 8.9 & 9 & 9 \\
\hline$\ell_{g}$ & 1 & 2 & 5 & 7 \\
\hline$\sigma_{r}(\mathrm{GFM})(\mathrm{mb})$ & 65.36 & 95.03 & 201.6 & 251.1 \\
\hline$\sigma_{r}(\mathrm{FM})(\mathrm{mb})$ & 1.6 & 6.2 & 37.71 & 72.2 \\
\hline$\chi^{2}$ & $0.25 \times 10^{-4}$ & $0.12 \times 10^{-5}$ & $0.13 \times 10^{-4}$ & $0.25 \times 10^{-5}$ \\
\hline
\end{tabular}

TABLE 3: Parameters extracted from Regge Pole analysis for the elastic scattering of ${ }^{16} \mathrm{O}$ nucleus by the target nucleus ${ }^{64} \mathrm{Zn}$ at incident energy of $40,41,42.5$ and 43.5MeV.

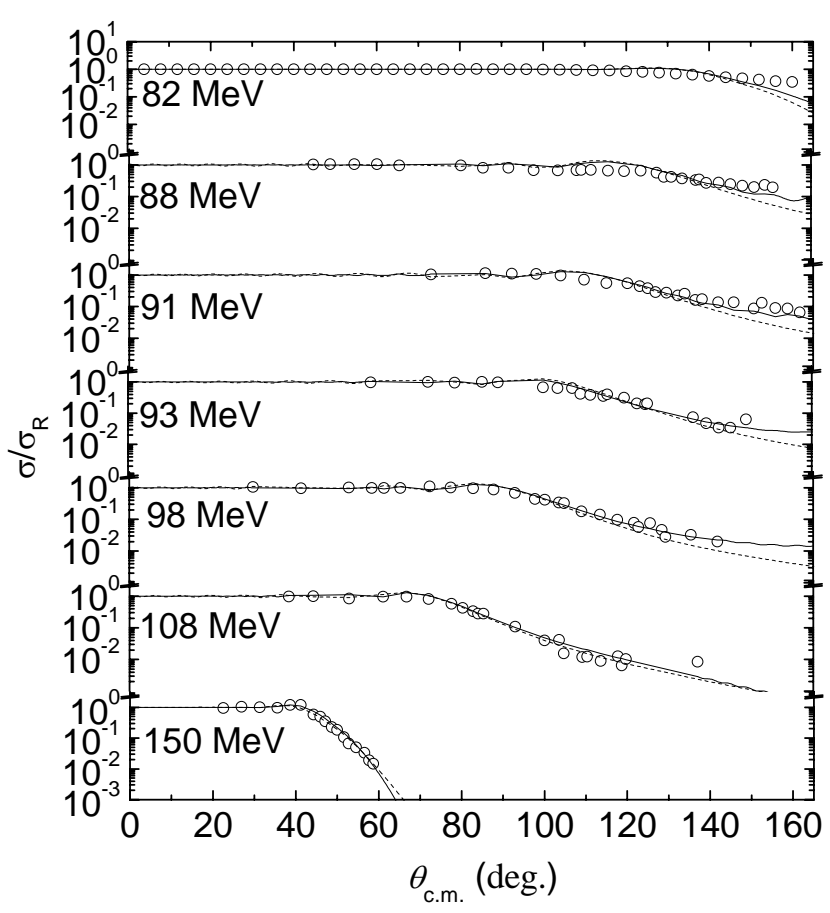

FIG. 6: Same as in Figure 4 but for fitting the experimental data of angular distribution (symbols) of elastic scattering ${ }^{32} \mathrm{~S}+{ }^{64} \mathrm{Ni}$ at $E_{l a b .}=82,88,91,93,98,108$ and $150 \mathrm{MeV}$ [21].

\begin{tabular}{|c|c|c|c|c|c|c|c|}
\hline$E_{\text {lab. }}(\mathrm{MeV})$ & 82 & 88 & 91 & 93 & 98 & 108 & 150 \\
\hline$\Lambda$ & 12.0 & 16.0 & 20.0 & 25.0 & 31.0 & 42.0 & 70.0 \\
\hline$\Delta$ & 2.0 & 0.1 & 0.2 & 0.4 & 0.4 & 0.6 & 3.5 \\
\hline$\mu(\mathrm{rad})$ & 0.1 & 0.1 & 0.2 & 0.2 & 0.2 & 0.5 & 2.5 \\
\hline$d(\mathrm{fm})$ & 0.070 & 0.005 & 0.011 & 0.026 & 0.030 & 0.052 & 0.314 \\
\hline$R(\mathrm{fm})$ & 12.016 & 11.372 & 11.210 & 11.280 & 11.165 & 11.118 & 10.867 \\
\hline$h$ & 1.018 & 1.034 & 1.054 & 1.084 & 1.131 & 1.241 & 1.685 \\
\hline$\theta_{R}(\mathrm{rad})$ & 2.610 & 2.422 & 2.250 & 2.055 & 1.831 & 1.482 & 0.872 \\
\hline$\theta_{1 / 4}(\mathrm{rad})$ & --- & 2.537 & 2.284 & 2.116 & 1.859 & 1.501 & 0.846 \\
\hline$\sigma_{r}(\mathrm{GFM})(\mathrm{mb})$ & 115.5 & 135.9 & 207.0 & 320.4 & 464.5 & 775.9 & 1671 \\
\hline$\sigma_{r}(\mathrm{FM})(\mathrm{mb})$ & 81.1 & 134.3 & 202.9 & 310.2 & 452.6 & 753.9 & 1508 \\
\hline$\chi^{2}$ & 1.99 & 16.5 & 9.3 & 6.5 & 6.8 & 5.9 & 2.4 \\
\hline$\Lambda(\mathrm{GFM})$ & 12.0 & 16.0 & 20.0 & 24.0 & 31.0 & 42.0 & 72.0 \\
\hline$\Delta(\mathrm{GFM})$ & 2.0 & 0.1 & 0.2 & 0.3 & 0.3 & 0.5 & 3.5 \\
\hline$\chi^{2}(\mathrm{GFM})$ & 2.3 & 22.2 & 16.7 & 18.8 & 7.2 & 9.3 & 2.6 \\
\hline
\end{tabular}

Table 4:Same as in Table 2, but for the elastic scattering ${ }^{32} \mathrm{~S}+{ }^{64} \mathrm{Ni}$ at $E_{\text {lab. }}=82,88,91$, 93, 98, 108 and $150 \mathrm{MeV}$ [21].

emergence of Fraunhofer oscillations, especially in the illuminated region when the energy increases. However, some mild Fraunhofer oscillations are damped by the Coulomb interaction and started to appear in the shadow region for energies 185 and $220 \mathrm{MeV}$. The value of the parameter $\Lambda$ increases with the increase in energy. Both the experimental data and theoretical calculation show that $\frac{\sigma}{\sigma_{R}}$ falls off exponentially at angles $\theta>\theta_{R}$ and at all energies. This is an indication for the dominance of Fresnel diffraction in this shadow region. Moreover the whole features of angular distribution are dominated by Fresnel scattering since the value of pattern parame- 


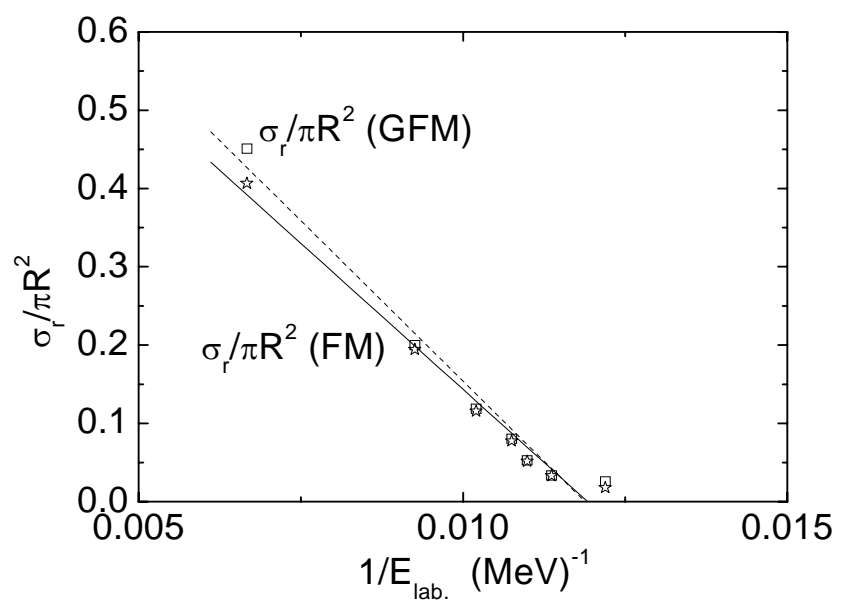

FIG. 7: Same as in Figure 5, but for the elastic scattering ${ }^{32} S+{ }^{64} \mathrm{Ni}$.

ter $p$, which is another measure for the degree of Fresnel-type diffraction, is very much larger than unity. Unfortunately, the values of parameters $\Lambda, \Delta, d$ and $\mu$ obtained from analysis of Frahn-Venter model are not consistent with the corresponding values of parameters obtained from McIntyre model [14] for this scattering. The ratio $d / R$ which provides the measure of Coulomb damping of Fraunhofer oscillations in the shadow region, do not have a clear correlation with the change in energy. The value of $R=10.68 \pm 0.18 \mathrm{fm}$ are in a very good agreement with those obtained from different analysis [14]. The variation of ratio of total reaction cross-section to $\pi R^{2}$ with the inverse of incident energy as obtained from the generalized Fresnel model (GFM) has almost a linear relation, as shown in figure 9. Similar variation is also obtained from the Fresnel model (FM) but with a slightly different straight line slope.

\section{CONCLUSION}

The theoretical results of angular distribution of elastic scattering at different laboratory energies yield a good agreement with corresponding experimental data on the basis of strong absorption model. The best theoretical fits to elastic scattering data are obtained using three parameters, namely, the grazing angular momenta $\Lambda$, the angular momentum window width $\Delta$ and $\mu$, when Frahn-Venter model is used. Other theoretical fits to same experimental data are also obtained with two parameters ( $\Lambda$ and $\Delta$ ) only, using the generalised Fresnel model. The quality of agreement between experiment and theory, here, is much better when full formalism of Frahn and Venter is used. Some fittings of theoretical angular distribution to corresponding data are fairly improved especially at backward angles when Regge pole analysis is employed. The quality of the fits is tested by the $\chi^{2}$ indicator. It is interesting to note that our theoretical fits of alpha scattering reactions compare fairly well with corresponding fits obtained using the modified Coulomb Glauber model. The analysis also demonstrates that the Fresnel-type scattering dominates the strong Coulomb interaction. The value of interaction radius exhibits

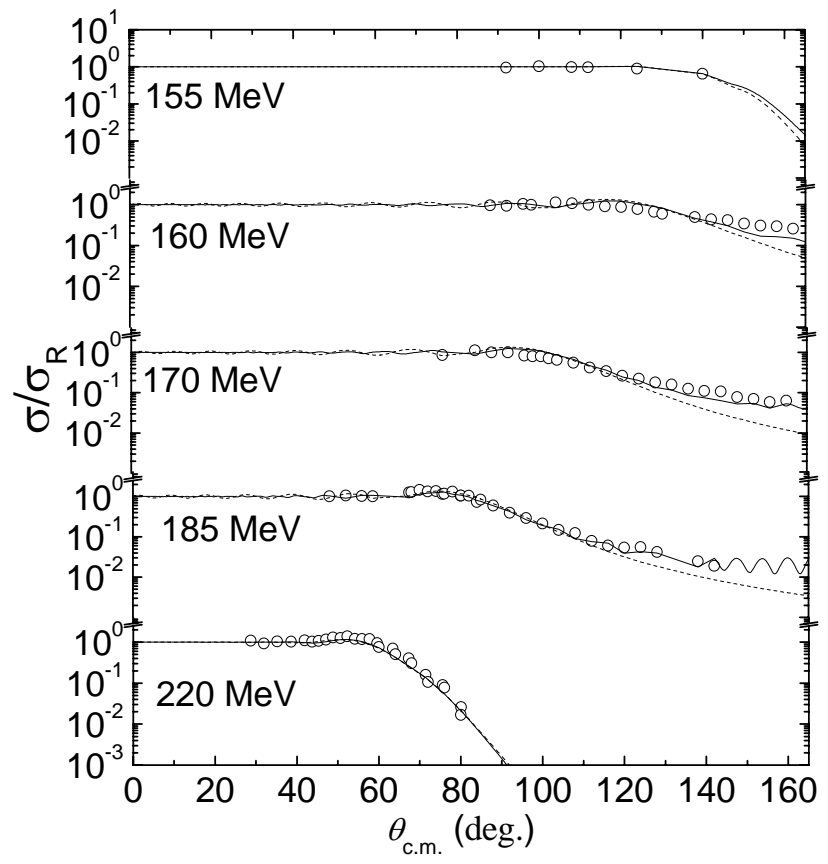

FIG. 8: Same as in Figure 4 but for fitting the experimental data of angular distribution (symbols) of elastic scattering ${ }^{58} \mathrm{Ni}+{ }^{27} \mathrm{Al}$ at $E_{\text {lab. }}=155,160,170,185$ and $220 \mathrm{MeV}$ [20].

\begin{tabular}{|c|c|c|c|c|c|}
\hline$E_{\text {lab. }}(\mathrm{MeV})$ & 155 & 160 & 170 & 185 & 220 \\
\hline$\Lambda$ & 10.0 & 11.0 & 20.0 & 29.0 & 44.0 \\
\hline$\Delta$ & 3.2 & 0.1 & 0.2 & 0.2 & 2.5 \\
\hline$\mu(\mathrm{rad})$ & 0.1 & 0.1 & 0.1 & 0.2 & 1 \\
\hline$d(\mathrm{fm})$ & 0.133 & 0.0045 & 0.015 & 0.019 & 0.265 \\
\hline$R(\mathrm{fm})$ & 10.858 & 10.569 & 10.506 & 10.470 & 10.495 \\
\hline$h$ & 1.020 & 1.025 & 1.082 & 1.174 & 1.399 \\
\hline$\theta_{R}(\mathrm{rad})$ & 2.586 & 2.525 & 2.065 & 1.672 & 1.179 \\
\hline$\theta_{1 / 4}(\mathrm{rad})$ & --- & 2.827 & 2.117 & 1.710 & 1.204 \\
\hline$\sigma_{r}(\mathrm{GFM})(\mathrm{mb})$ & 143.71 & 86.4 & 269 & 517 & 1110 \\
\hline$\sigma_{r}(\mathrm{FM})(\mathrm{mb})$ & 72.37 & 84.8 & 264 & 510 & 987 \\
\hline$\chi^{2}$ & 0.35 & 5.1 & 10.4 & 3.1 & 5.2 \\
\hline$\Lambda(\mathrm{GFM})$ & 9.8 & 11.0 & 20.0 & 28.0 & 44.0 \\
\hline$\Delta(\mathrm{GFM})$ & 3.2 & 0.1 & 0.2 & 0.2 & 2.5 \\
\hline$\chi^{2}(\mathrm{GFM})$ & 0.39 & 11.2 & 16.7 & 2.3 & 2.9 \\
\hline
\end{tabular}

Table 5: Same as in Table 2, but for the elastic scattering ${ }^{58} \mathrm{Ni}+{ }^{27} \mathrm{Al}$ at $E_{\text {lab. }}=155,160,170,185$ and $220 \mathrm{MeV}[20]$.

a gradual decrease as the energy increases in all elastic scattering processes, as expected from the prescriptions of SAM. The extracted parameters using the Frahn-Venter model can be correlated to the three-McIntyre parameters in the parameterized scattering matrix elements in a crude approximation; i.e. $\Lambda \approx \ell_{g}+\frac{1}{2}, \Delta \approx \Delta_{M}$ and $\mu_{M} \approx \frac{\mu}{2 \Delta}$. Some failures may be noted when the incident energy approaches the Coulomb barrier. The three-McIntyre parameters can be found and used as an input to a parameterised DWBA calculation of a trans- 


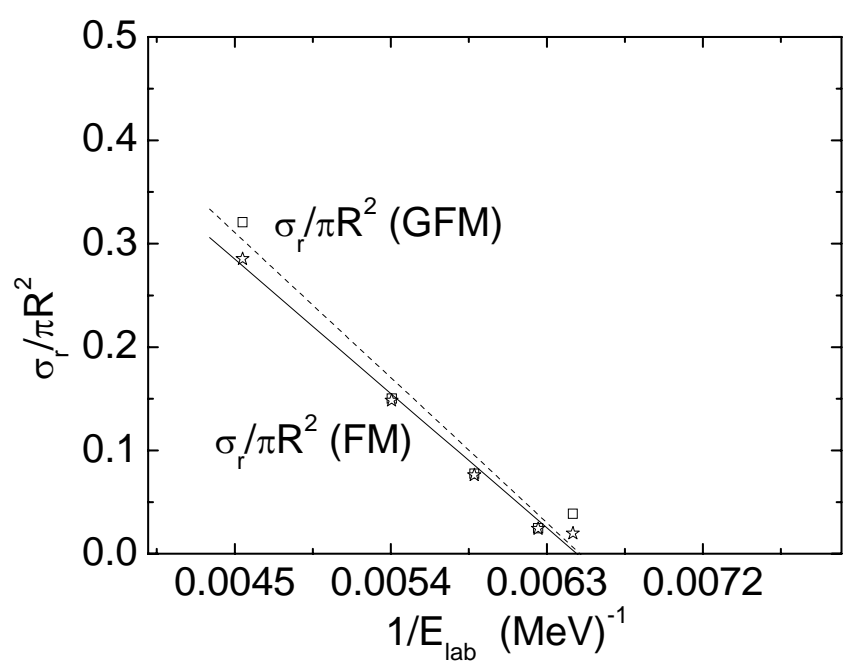

FIG. 9: Same as in Figure 5, but for the elastic scattering ${ }^{58} \mathrm{Ni}+{ }^{27} \mathrm{Al}$. fer reaction that has an incoming (or outgoing) energy in the entrance (or exit) channel using the diffraction model.

By comparing the values of $(n, h)$ or $(\Lambda, p)$ among all chosen elastic scattering processes at different energies, no "homomorphic" cases are found because none of these processes display identical diffraction patterns. Moreover, no glory or orbiting effect does exist in the structures of angular distribution for these elastic scattering processes at different energies. Unsuccessful fittings could be attributed to weak absorption in some of elastic scattering at certain energies; while the possibility of depletion of measured cross-sections to other open channels still exist in some cases. However, any of the obtained theoretical results and experimental data do not have any broad structures at large angles that can be attributed to an Airy interference pattern.
[1] R. H. Venter, Ann. Phys. 25 (1963) 405

[2] W. E. Frahn and R. H. Venter, Ann. Phys. 24 (1963) 243.

[3] W. E. Frahn and K. E. Rehm, phys Rep. (Sect. C of Phys. Lett.) 37 (1987) 1

[4] W. E. Frahn, Ann. Phys. 72 (1972) 524.

[5] W. E. Frahn, Nucl. Phys. 75 (1966) 577.

[6] W. E. Frahn, Nucl. Phys. A 302 (1978) 267.

[7] J. A. McIntyre, K. H. Wang and L. C. Becker, Phys. Rev. 117 (1960) 1337.

[8] J. A. McIntyre, S. D. Baker and K. H. Wang, Phys. Rev. 125 (1962) 584.

[9] M. C. Mermaz, B. Bilwes, R. Bilwes and J. L. Ferrero, Phys. Rev. C27 (1983) 2408.

[10] M. C. Mermaz Z. Phys. A: Atoms and Nuclei, 321 (1985) 613.

[11] R. I. Badran, I. M. Naqib, D. J. Parker and J. Asher, J. Phys. G, 22 (1996) 1441.

[12] R. I. Badran, D. J. Parker, I. M. Naqib, Euro. J. Phys. A12 (2001) 1 .

[13] R. I. Badran, Arab. J. Sci. Eng., 27 (2002) 65.

[14] R. I. Badran, APH N. S., Heavy ion Physics 17/1 (2003) 151.

[15] L. N. Pandey and S. N. Mukherjee, Phys. Rev. C29 (1984) 1326.

[16] M. H. Cha, B. K. Lee and Y. J. Kim, J. Kor. Phys. Soc. 31
(1997) 360.

[17] H. L. Clark, Y. W. Lui and D. H. Youngblood, Nucl. Phys. A 589 (1995) 416.

[18] M. A. Alvi, J. H. Madani and A. M. Hakmi Phys. Rev. C 75 (2007) 064609-1.

[19] C. Tenreiro, J. C. Acquadro, P. A. B. Freitas, R. Liguori Neto, G. Ramirez, N. Cuevas, P. R. S. Gomes , R. Cabezas, R. M. Anjos, and J. Copne, Phys. Rev. C 53, (1996) 2870.

[20] M. E. Brandan, J. R. Alfaro, A. Menchaca-Rocha, J. Gmez del Campo, G. R. Satchler, P. H. Stelson, H. J. Kim, and D. Shapira, Phys. Rev. C 48 (1993) 1147.

[21] A. M. Stefanini, D. Bonamini, A. Tivelli, G. Montagnoli G., Fortuna, Y. Nagashima, S. Beghini, C. Signorini, A. DeRosa, G.Inglima, M. Sandoli, G. Cardella, and F. Rizzo, Phys. Rev. Lett. 59 (1987) 2852.

[22] M. C. Mermaz, E. R. Chavez-Lomeli, J. Berthier, and A. Greiner, Phys. Rev. C 29 (1984) 147-157.

[23] G. Igo, H. E. Wegner and R. M. Eisberg, Phys. Rev. 101 (1956) 1508.

[24] D. D. Kerlee, J. S. Blair and G. W. Farwell, Phys. Rev. 107 (1957) 1343. 